\title{
Colocación de clavo retrógrado humeral: reporte de un caso
}

\author{
Humeral retrograde nailing: a case report \\ Jonathan R Hernández-Molina, * Alejandra López-Rubio, ${ }^{\ddagger}$ Alicia Villalobos-Rodríguez $\$$ \\ ${ }^{*}$ Titular adjunto del Curso de Cirugía Articular; ${ }^{\ddagger}$ Residente de $4^{\circ}$ año en Traumatología y Ortopedia; $\$$ Médico adscrito al \\ Departamento de Cirugía Articular de Hombro y Rodilla. \\ Hospital Ángeles Mocel. México.
}

\begin{abstract}
Resumen
El tratamiento de las fracturas diafisarias del húmero continúa siendo controversial. El enclavado centromedular humeral (ECH) se perfila como el estándar de oro para el tratamiento de este tipo de fracturas. En la técnica anterógrada, el acceso permite entrar de forma directa a la epífisis humeral proximal, con la desventaja de involucrar una articulación sana, generando dolor crónico o la necesidad de cirugías de revisión. La técnica retrógrada elimina el riesgo de dolor de hombro postoperatorio, lesión del manguito rotador y lesión del nervio axilar. Es más compleja, ya que el sitio de entrada es una capa delgada de hueso, generando como complicación principal una fractura iatrogénica. Presentamos a masculino de 28 años, atropellado en vía pública, con mecanismo de contusión directa en brazo izquierdo. Inicia con dolor severo, deformidad, aumento de volumen y pérdida de la función, acudiendo al Servicio de Urgencias del Hospital Ángeles Mocel para valoración y tratamiento. Radiográficamente con fractura simple transversa en tercio medio del húmero. Se somete a tratamiento definitivo con $\mathrm{ECH}$ retrógrado. Al séptimo día postquirúrgico presenta arcometría completa para hombros y codos, con dolor únicamente en zona de abordaje.
\end{abstract}

Palabras clave: Húmero, enclavado centromedular, retrógrado.

\begin{abstract}
The treatment of shaft fractures of the humerus to this day is still controversial. Humeral intramedullary nailing (HIN) is emerging as the gold standard for the treatment of this type of fracture. In the antegrade technique, the access allows direct entry to the proximal humerus epiphysis, with the disadvantage of involving a healthy joint, generating chronic pain or the need for revision surgeries. The retrograde technique eliminates the risk of postoperative shoulder pain, rotator cuff injury and axillar nerve injury. It is more complex since the entry point is a thin layer of bone, generating an iatrogenic fracture as the main complication. We present a 28-year-old male, run over, with a direct contusion on the left arm. He began with severe pan, deformity, increased volume and loss of function, going to the emergency department at Hospital Ángeles Mocel for evaluation and treatment. Radiographically with a simple transverse fracture in the middle third of the humerus. He undergoes definitive treatment with retrograde HIN. On the seventh postoperative day, he presents full range of motion in shoulders and elbows, with pain in the approach area.
\end{abstract}

Keywords: Humerus, intramedullary nailing, retrograde.

\section{Introducción}

El tratamiento de las fracturas diafisarias del húmero continúa siendo controversial. Diversos autores favorecen la utilización de placa, mientras que otros prefieren la utilización de un clavo endomedular. ${ }^{1-3}$ Gracias al estudio de diversas fundaciones como AO trauma, el enclavado centromedular (EC) se perfila como el estándar del tratamiento para este tipo de fracturas, debido a que disminuye la manipulación de partes blandas, evitando la desperiostización excesiva del hueso y consiguiendo una consolidación biológica con formación de callo óseo. ${ }^{1,4}$

Correspondencia:

Jonathan R Hernández-Molina

E-mail: jonyhm@hotmail.com

Recibido: 26-08-2021. Aceptado: 04-10-2021.

Citar como: Hernández-Molina JR, López-Rubio A, Villalobos-Rodríguez A. Colocación de clavo retrógrado humeral: reporte de un caso. Orthotips. 2022; 18 (1): 68-71. https://dx.doi.org/10.35366/103735 
Existen dos técnicas para el EC, la anterógrada fue la primera en describirse y es técnicamente más sencilla, dado que el acceso quirúrgico permite acceder de forma directa a la epífisis humeral proximal. ${ }^{5,6} \mathrm{Sin}$ embargo, involucra una articulación sana generando a largo plazo dolor crónico o la necesidad de cirugías de revisión. ${ }^{2,6}$ Por otro lado, el enclavado centromedular retrógrado $(E C R)$ elimina el riesgo de dolor de hombro postoperatorio, lesión del manguito rotador y del nervio axilar, al no involucrar ninguna articulación. ${ }^{1,7}$ Sin embargo, es más compleja, debido a que el sitio de entrada es una capa delgada de hueso, teniendo como complicación principal una fractura conminuta del húmero distal. ${ }^{3,8-10}$ El objetivo del trabajo es presentar un caso clínico exitoso de ECR en húmero.

\section{Presentación del caso}

Cuadro clínico. Masculino de 28 años con antecedente de atropellamiento en vía pública, con mecanismo de contusión directa en brazo izquierdo. Inicia con dolor severo, deformidad, aumento de volumen y pérdida de la función, acudiendo al Servicio de Urgencias del Hospital Ángeles Mocel para valoración y tratamiento. Radiográficamente presenta trazo simple transverso en tercio medio del húmero (Figura 1A). Se somete a tratamiento definitivo con ECR, la cual ocurre sin complicaciones (Figura 1B). Al séptimo día postquirúrgico presenta arcos de movimiento completos para hombros y codos, con dolor únicamente en zona de abordaje (Figura 2).

Técnica quirúrgica. Se colocó al paciente en decúbito prono con el brazo izquierdo en abducción a $90^{\circ}$ sobre un bulto, con la capacidad para lograr

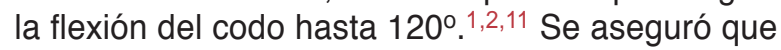
el fluoroscopio diera las imágenes adecuadas en anteroposterior (AP) y lateral, así como húmero distal y proximal. La longitud del clavo se puede deducir previo a la cirugía midiendo el húmero contralateral, o bajo visión fluoroscópica con la regla del equipo una vez que haya iniciado la cirugía. ${ }^{12,13}$ De la misma manera, se puede calcular el ancho del clavo, que se debe medir en el istmo del canal medular.

Con el codo en flexión de $90^{\circ}$, se realizó una incisión distal al olécranon y se incidió en el tríceps. ${ }^{1,2,7}$ Al llegar a la diáfisis se localizó una sección de hueso en el borde superior de la fosa olecraneana, $40 \mathrm{~mm}$ superior a este borde es donde se realiza la entrada del clavo. Hay que asegurarse de que se esté justo en la línea media, ya que lateralizar el punto de entrada provocaría mucho estrés en la cortical lateral.,2,11 Se aperturó el canal medular con broca de $4.5 \mathrm{~mm}$, creando un triángulo con base distal (Figura 3). Posteriormente, utilizando una fresa con punta, se hizo la entrada de $2 \times 1 \mathrm{~cm}$, y con la ayuda de la fresa cilíndrica se le dio forma al punto de entrada. Procedimos a la inserción del clavo endomedular. Se recomienda no forzar la entrada del clavo con martillo, ya que esto puede provocar fractura en el sitio de entrada. ${ }^{1,14}$ En algunas ocasiones la estrechez del ángulo dificulta la entrada, en nuestro caso se tuvo que utilizar el martillo con mucho cuidado ya que, a pesar de usar el clavo más pequeño, tuvimos dificultad para adentrarlo. Esto se hizo bajo visión fluoroscópica, asegurándonos de no fracturar el sitio de entrada.

Se avanzó el clavo hasta que la punta estuviera dentro de la cabeza humeral. ${ }^{2}$ Se recomienda que si
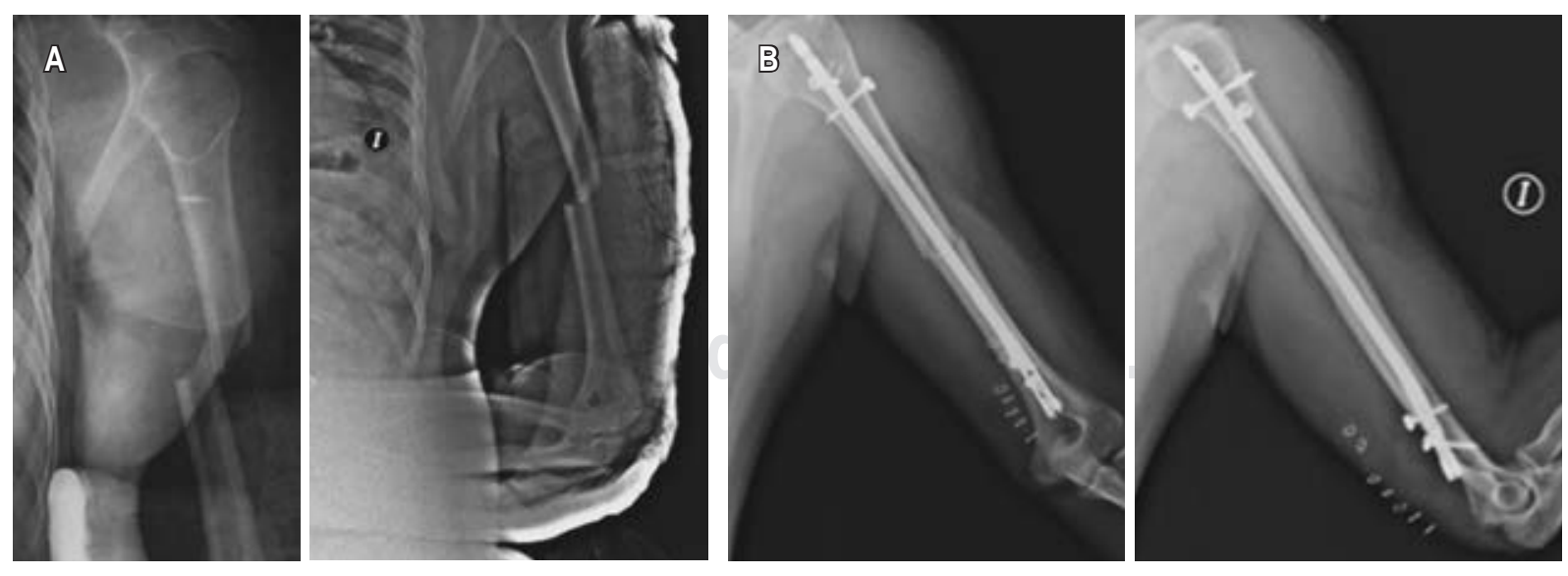

Figura 1: Radiografías anteroposteriores (AP) y laterales del húmero izquierdo. A) Prequirúrgico: muestra trazo simple transverso en tercio medio del húmero. B) Postquirúrgico: adecuada reducción y colocación de implante centromedular en húmero. 

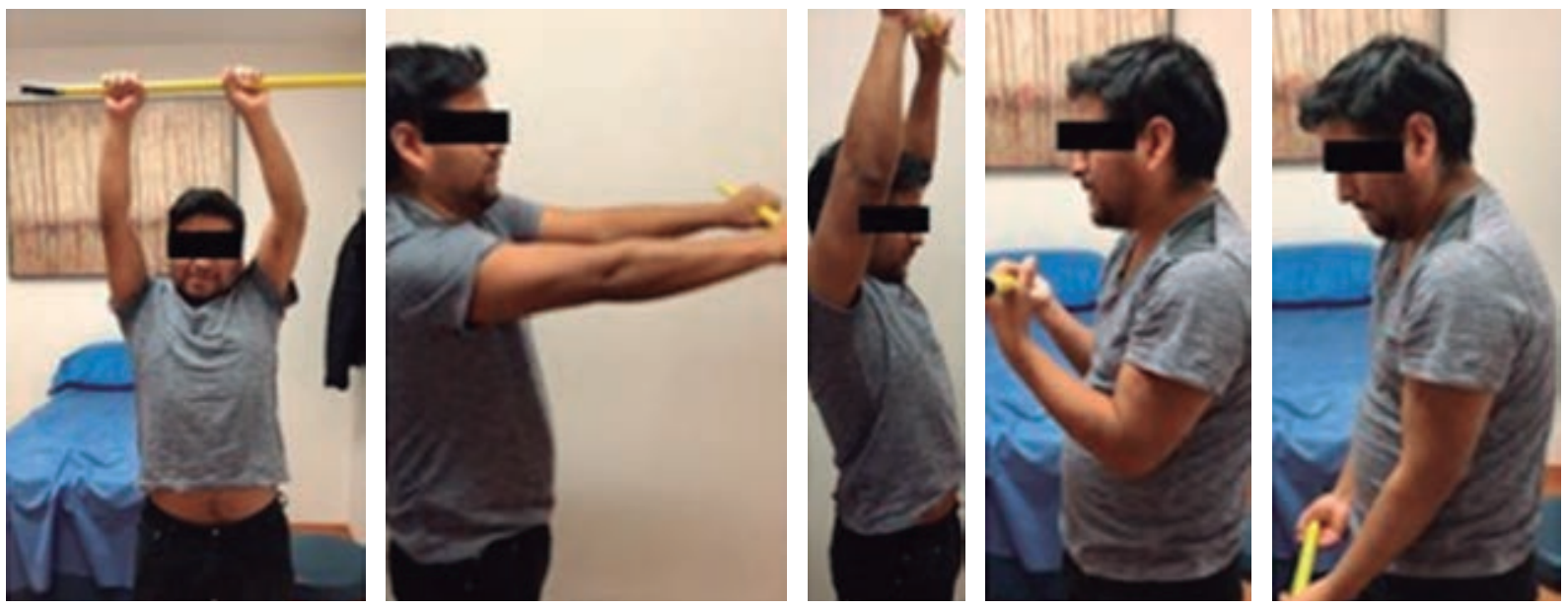

Figura 2: Siete días postquirúrgicos: arcometría completa de hombros y codos.

se reduce bien el sitio de fractura, se pueden colocar primero los tornillos proximales, bajo visión fluoroscópica. $8,15,16$ Hay que tomar en cuenta el recorrido del nervio axilar, aproximadamente $5.5 \mathrm{~cm}$ debajo del acromion para no dañarlo durante la inserción de los tornillos. ${ }^{2,3}$ Para el bloqueo distal existe una guía que facilita la colocación de los tornillos. Nosotros colocamos primero los tornillos distales, utilizando el orificio dinámico. Por último, colocamos la tapa en el extremo distal del clavo. Esto evita desplazamiento del clavo, así como crecimiento de hueso y tejido dentro del clavo y facilita su extracción en caso de ser necesario. ${ }^{15,17}$ Postquirúrgicamente sólo se evitan movimientos rotacionales del brazo con resistencia, arcos de movimiento de hombro y codo libre.

Si existe un trazo transverso u oblicuo corto es recomendable realizar compresión a través del clavo endomedular. ${ }^{3,4,18} \mathrm{Si}$ se planea hacer compresión, el clavo se tiene que meter más milímetros de lo anticipado, para que al realizarla el clavo no se exponga en su sitio de entrada, la distancia máxima es de 8 $\mathrm{mm} \cdot{ }^{3,19}$ Se finaliza colocando el tornillo de compresión al clavo, al aplicarlo hace que el fragmento proximal se mueva en dirección distal. Para asegurarlo hay que colocar el tornillo en el agujero estático. Si el paciente tiene lesión del nervio radial prequirúrgicamente, se recomienda hacer una exploración transoperatoria con una incisión anterolateral. ${ }^{1,2}$

\section{Discusión}

El uso del ECR en este caso consiguió buenos resultados a corto plazo. Las indicaciones para dicho

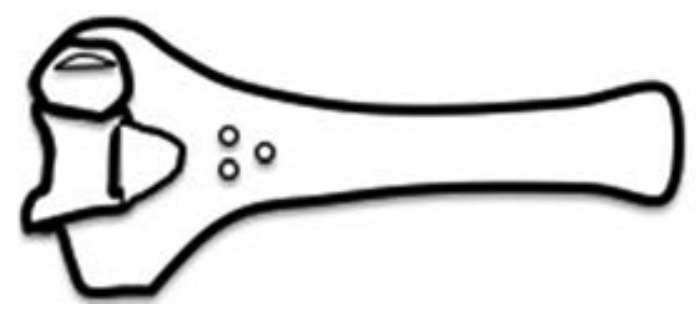

Figura 3: Triángulo con base distal para entrada del enclavado centromedular.

implante incluyen fracturas en el tercio medio del húmero, como nuestro paciente, así como fracturas expuestas, lesión nerviosa, retraso en la unión, entre otras. ${ }^{5}$ En cuanto a la técnica, colocamos al paciente en decúbito prono $0^{1,5}$ con la habilidad de flexionar el codo a casi los $120^{\circ} .4,5$ La principal ventaja que ofrece esta técnica es no afectar una articulación como se hace con el enclavado anterógrado. ${ }^{20}$ La colocación de dicho implante requiere destreza por parte del cirujano, ya que requiere conocimiento anatómico y de la técnica precisa. El no conocer cualquiera de las dos anteriores, puede dar resultados fatales que disminuyen las ventajas del enclavado centromedular. ${ }^{6,14}$ Basándonos en la literatura, este paciente fue elegido adecuadamente para la colocación del ECR y se hizo sin incidentes, por lo que se espera que la recuperación del paciente sea mucho más rápida y pueda volver a sus actividades de manera temprana. ${ }^{8}$

\section{Conclusiones}

Actualmente, la vía retrógrada está acaparando la atención del ortopedista como tratamiento de la 
fractura diafisaria humeral, gracias a los resultados altamente satisfactorios y disminución de costos asociados. Los cirujanos cada vez se entrenan más en esta técnica y poco a poco vamos viendo más y mejores resultados. Se debe elegir al paciente adecuado para este tipo de implante endomedular para obtener resultados satisfactorios.

\section{Referencias}

1. Yi JW, Lee JS, Cho HJ. Retrograde intramedullary nailing for humerus fracture in a supine position: performing an unfamiliar procedure in a familiar position. Clin Orthop Surg. 2017; 9 (3): 392-395.

2. Morales Santos G, Salazar Pacheco R. Tratamiento de fracturas del húmero con clavo centromedular no rimado. Rev Hosp Jua Mex. 2001; 68 (4): 178-186.

3. Heineman DJ, Poolman RW, Nork SE, Ponsen KJ, Bhandari M. Plate fixation or intramedullary fixation of humeral shaft fractures. Acta Orthop. 2010; 81 (2): 216-223.

4. Biber R, Zirngibl B, Bail HJ, Stedtfeld HW. An innovative technique of rear entry creation for retrograde humeral nailing: how to avoid iatrogenic comminution. Injury. 2013; 44 (4): 514-517.

5. Hollister AM, Saulsbery C, Odom JL, Anissian L, Garon MT, Jordan J. New technique for humerus shaft fracture retrograde intramedullary nailing. Tech Hand Up Extrem Surg. 2011; 15 (3): 138-143.

6. Lanting B, MacDermid J, Drosdowech D, Faber KJ. Proximal humeral fractures: a systematic review of treatment modalities. J Shoulder Elbow Surg. 2008; 17 (1): 42-54.

7. Pidhorz L. Acute and chronic humeral shaft fractures in adults. Orthop Traumatol Surg Res. 2015; 101 (1 Suppl): S41-S49.

8. Browner B, Jupiter J, Krettek C, Anderson P. Fracture of the humeral shaft. Skeletal trauma. Philadelphia, PA: Elsevier; 2015. pp. 1389-1405.

9. Kumar R, Singh P, Chaudhary LJ, Singh S. Humeral shaft fracture management, a prospective study; nailing or plating. J Clin Orthop Trauma. 2012; 3 (1): 37-42.
10. Strohm PC, Reising K, Hammer T, Sudkamp NP, Jaeger M, Schmal $\mathrm{H}$. Humerus shaft fractures - where are we today? Acta Chir Orthop Traumatol Cech. 2011; 78 (3): 185-189.

11. Baltov A, Mihail R, Dian E. Complications after interlocking intramedullary nailing of humeral shaft fractures. Injury. 2014; 45 Suppl 1: S9-S15.

12. Verdano MA, Pellegrini A, Schiavi P, Somenzi L, Concari G, Ceccarelli $F$. Humeral shaft fractures treated with antegrade intramedullary nailing: what are the consequences for the rotator cuff? Int Orthop. 2013; 37 (10): 2001-2007.

13. Benegas E, Ferreira Neto A, Oliveira Marchitto G. Humeral shaft fractures. Rev Bras Ortop. 2010; 45 (1): 12-16.

14. Ruffilli A, Traina F, Pilla F, Fenga D, Faldini C. Marchetti Vicenzi elastic retrograde nail in the treatment of humeral shaft fractures: review of the current literature. Musculoskelet Surg. 2015; 99 (3): 201-209.

15. Sharma GM, Bhardwaj AR, Shah S. Antegrade versus retrograde nailing in humeral shaft fractures: a prospective study. J Clin Orthop Trauma. 2020; 11 (Suppl 1): S37-S41.

16. Bencic I, Cengic T, Prenc J, Bulatovic N, Matejcic A. Humeral nail: comparison of the antegrade and retrograde application. Acta Clin Croat. 2016; 55 (1):110-116.

17. Updegrove GF, Mourad W, Abboud JA. Humeral shaft fractures. J Shoulder Elbow Surg. 2018; 27 (4): e87-e97.

18. Schoch BS, Padegimas EM, Maltenfort M, Krieg J, Namdari S. Humeral shaft fractures: national trends in management. $J$ Orthop Traumatol. 2017; 18 (3): 259-263.

19. Westrick E, Hamilton B, Toogood P, Henley B, Firoozabadi R. Humeral shaft fractures: results of operative and non-operative treatment. Int Orthop. 2017; 41 (2): 385-395.

20. Dilisio MF, Nowinski RJ, Hatzidakis AM, Fehringer EV. Intramedullary nailing of the proximal humerus: evolution, technique, and results. J Shoulder Elbow Surg. 2016; 25 (5): e130-e138.

\section{Conflicto de intereses}

Los autores declaramos que no tenemos ningún conflicto de intereses para este trabajo. 